\title{
La ville entre les lignes de la science et du roman
}

Pierre LASSAVE

"Les hommes ne savent pas comment le différent concorde avec luimême ; il est une harmonie contretendue comme pour l'arc et la lyre".

Héraclite

\footnotetext{
D
} ans la civilisation occidentale, la ville au sens général a souvent aidé les écrivains et les philosophes à exprimer et à penser leur temps. Lorsqu'il voit dans la ville davantage qu'un simple décor de l'intrigue, le roman contemporain éprouve la puissance métaphorique de la Cité. Celle-ci devient par exemple labyrinthe quand ses formes trop envahissantes pour être visibles ne retiennent plus une mémoire, ni une identité. Les figures alarmantes de la métropole industrielle d'hier - machine infernale, monstre tentaculaire, jungle humaine - se dissolvent alors dans l'énigme du présent urbanisé. L'histoire de la littérature nous rappelle le trésor mythologique accumulé par la ville depuis l'Antiquité (KERBRAT, 1995). De la Genèse à l'Encyclopédie des Lumières en passant par les Utopies de More ou de Campanella, la légende urbaine regorge d'antinomies : entre le divin et l'humain (Jérusalem Céleste / Jérusalem Terrestre), entre la splendeur et la misère d'une civilisation opposée à la barbarie, etc. Enceinte carrée et tracée dans le sang sacrificiel face à l'inatteignable cosmos circulaire, la Cité est devenue le temple de la loi humaine, celle qui asservit autant que celle qui libère. A la croisée des 
chemins, elle capte le nomade et affranchit l'étranger de son altérité menacante ; sa polarité géographique comme son œuvre architecturale marquent au sol la mémoire du contrat social par lequel la modernité advient, du parlement au marché.

Cependant, du Roman de Renart au dernier polar d'aujourd'hui, le genre romanesque dans sa diversité s'est développé en retrait et en contrepoint des grands récits mythiques ou philosophiques. On a attribué le succès du roman moderne depuis Don Quichotte ou Robinson Crusoé au fait qu'il a célébré l'individualité (écrivain, héros et lecteur) et qu'il a rempli une fonction d'identification sociale en remettant en cause les ordres établis (ZÉRAFFA, 1971). Lorsqu'à l'ère industrielle la science a pris le relais de la vieille métaphysique, le romancier s'est voulu "secrétaire de la Société" (Balzac, Avant-propos de 1842 à La Comédie humaine) puis "révélateur de la nature humaine" (Zola, Le roman expérimental, 1880) sur le modèle neutre et objectif d'une expérience scientifique. Professions de foi naturalistes qui n'ont pas plus résisté à la transfiguration du réel par ces deux grands auteurs qu'à la critique du dogme réaliste dès la fin du XIX ${ }^{\text {eme }}$ siècle. Au moment même d'ailleurs où les sciences humaines naissantes prétendaient au monopole de l'expression rationnelle du monde social, renvoyant ainsi le roman à son lieu esthétique. Division des genres qui a imposé ses oppositions formelles telles que vérité et fiction, objectivité et subjectivité, raison et sentiment, etc. A y regarder de près, les choses sont évidemment moins nettes et au terme de ce siècle les frontières entre science et littérature se font plus poreuses.

Plus que jamais conscientes de leur relativité fondamentale ou de leur destin assertorique, les sciences sociales ou de la culture s'interrogent aujourd'hui sur leur propre fonctionnement littéraire'. La question de la narration s'immisce au sein du dialogue entre les concepts et les données, entre la description et l'explication du monde. L'anthropologue introduit le Récit au beau milieu de son entretien forcé entre Soi et l'Autre. Les Tristes tropiques vibrent encore de leurs multiples cordes sensibles : récit de voyage aux antipodes, pamphlet contre l'ethnocide, méditation sur le contrat social, thèse structuraliste, hymne au symbolisme terrestre, etc. (GEERTZ, 1988). L'historien de son côté est sollicité pour reconnaître la "poétique" de son savoir, c'est-à-dire mettre à jour les procédures proprement littéraires par lesquelles son discours se soustrait à la fiction et autoproclame sa science (RANCIËRE, 1992). Le sociologue, quant à lui, retrouve les "clefs" de son étrange poétique (BROWN, 1977) et fait son

1. On peut en prendre la mesure dans le dossier bien documenté " $L$ 'écriture des sciences de l'homme" du numéro 58 de Communications (1994) 
Iger de son altérité œuvre architecturale la modernité advient,

'aujourd'hui, le genre et en contrepoint des lé le succès du roman u fait qu'il a célébré rempli une fonction is établis (ZÉRAFFA, e relais de la vieille : la Société" (Balzac, évélateur de la nature odèle neutre et objectif istes qui n'ont pas plus uuteurs qu'à la critique snt même d'ailleurs où copole de l'expression à son lieu esthétique. :lles telles que vérité et c. A y regarder de près, : ce siècle les frontières

rentale ou de leur destin rrogent aujourd'hui sur narration s'immisce au entre la description et Récit au beau milieu de iques vibrent encore de ux antipodes, pamphlet se structuraliste, hymne storien de son côté est 'est-à-dire mettre à jour discours se soustrait à la b). Le sociologue, quant :OWN, 1977) et fait son miel du signifié romanesque par delà l'examen des relations entre couvre, institution littéraire et public ${ }^{2}$. Bien que sévèrement élevée dans la résistance à la tentation littéraire (LEPENIES, 1985), cette dernière discipline semble aujourd'hui en mesure de reconnaître dans La Recherche de Proust l'une de ses plus pénétrantes analyse du remplacement de la maison aristocratique par le salon bourgeois (BIDOU-ZACHARIASEN, 1997) . En parallèle à ces glissements épistémiques, la critique littéraire s'écarte du champ proprement sémiotique et commence à voir dans certaines cuvres les recherches les plus pénétrantes sur la vérité humaine (CAMPION, 1996). Non plus évidemment la vérité de l'illusion réaliste, mais celle du rapport problématique que l'homme entretient avec le monde vécu.

En définitive, si la confusion des genres entre science et littérature n'est sans doute pas pour demain tant la division des langues est au principe de la communication humaine depuis Babel, la reconnaissance de dettes réciproques de sens entre le roman et la sociologie semble se faire plus pressante. Dans cette perspective, la ville peut devenir un analyseur de choix de cet échange comme pourraient également l'être l'amour pour la psychologie et la mort pour la philosophie ou l'anthropologie. Ainsi qu'on l'a montré, la reconnaissance de la ville comme objet sociologique a cependant été rien moins qu'immédiate malgré le fait indéniable que l'urbanisation est un phénomène central de notre époque (LASSAVE,1997). Domaine de prédilection des géographes, le rapport que les sociétés entretiennent avec leur espace a en effet tardé en France à être systématiquement réfléchi par les sociologues, au moins sous son double aspect de produit et d'agent de l'organisation sociale et de l'identité individuelle (l'exception de l'œuvre momentanément isolée d'Halbwachs confirmant la règle). Il a paradoxalement fallu que sous 1'influence conjointe du marxisme et du structuralisme (dans la période 1965-75) la ville soit à la fois saisie et déniée comme objet théorique pour que le scepticisme naturel des sociologues la reconsidère comme laboratoire incontournable d'observation des formes de sociabilité. D'où la redécouverte complémentaire et des réflexions de Georg Simmel ou de Max Weber sur l'urbanité comme trait de civilisation et de l'Ecole de Chicago, expérience fondatrice de la sociologie urbaine à l'entre-deux-

2. Par exemple, les travaux récents de N. Heinich sur l'identité féminine (1996) ou de F. de Singly sur le couple (1996).

3. C. Bidou-Zachariasen voit dans l'œuvre de Proust moins l'illustration prestigieuse de la "sociologie littéraire" inspirée de Tarde que l'anticipation des thèses d'Elias sur la dynamique de l'Occident, la "configuration" (concept dynamique dépassant l'antinomie classique entre individu et société) ou le mixte d'engagement et de distance propre à l'observation ethnogra" phique contemporaine. 
guerres. Aujourd'hui emblême des politiques européennes de prévention des risques d'anomie sociale, la ville est devenue une métaphore centrale pour désigner la crise des Etats providence ou de la société salariale. Au-delà de ses manifestations variées que les sociologues s'attachent à décrire suivant les contextes locaux, le phénomène urbain ne résiste pas moins comme objet de connaissance anthropologique en soi.

Que ce soit donc à titre épistémologique (relations modales entre les récits $\mathrm{du}$ romancier et du sociologue) ou plus largement philosophique (l'interrogation sur les caractères de la modernité), l'exploration historique des apports réciproques de la littérature et de la sociologie à la connaissance du phénomène urbain (en tant que complexe spécifique d'attitudes et de mentalités) reste tout un programme ${ }^{4}$. On s'en tiendra ici pour notre part à l'évrcation de quelques cas de villes-époques ou "chronotopes", suivant l'expression de Mikhail Bakhtines, doublement soumis à la plume de l'écrivain et du savant sans que l'on puisse encore conclure sur leur caractère véritablement significatif.

Le choix de ces situations a d'abord été guidé par la connaissance acquise des moments marquants de la rencontre entre la sociologie universitaire et la ville. Ainsi, au double titre des prémices de l'enquête sociologique et du roman social dans la ville-machine de la première révolution industrielle a-ton retenu le jeune Friedrich Engels (Die Lage der arbeiten klassen in England) et le mûr Charles Dickens (Hard Times) au cœur du Pays Noir anglais, à Manchester précisément ; on ne peut échapper ensuite au laboratoire urbain de Chicago en rappelant deux textes fondateurs ou testamentaires (Die Grossstädte und das Geistesleben de G. Simmel et Urbanism as a Way of Life de L. Wirth) auxquels on confrontera Sister Carrie de Theodore Dreiser, pionnier du roman social américain, puis à New York, le fameux Manhattan Transfer de John Dos Passos, œuvre dont la forme kaléidoscopique représente remarquablement le destin métropolitain. L'entrée par la littérature plutôt que par la sociologie aurait pu certes conduire vers d'autres choix, par exemple le Paris de Balzac, puis le Vienne de Musil ou le Dublin de Joyce, mais on serait alors bien en peine de leur trouver quelques correspondants scientifiques directs. Enfin, au titre de la réflexion actuelle et pléthorique sur la ville d'aujourd'hui, on s'est orienté vers Marseille non seulement pour des raisons de circonstance (connaissance native des lieux, participation à un atelier local

4. C'est en tout cas celui de P. Saint-Arnaud (1998) qui compare systématiquement les points de vue du sociologue R.E. Park et du romancier J. Dos Passos sur la modernité urbaine ou le
malaise de la ville américaine au début du siècle.

5. Par cette expression, M. Bakhtine (1975) désignait précisément l'organisation essentielle de l'espace-temps dans le récit romanesque. Si la route est par exemple le chronotope typique du roman d'aventures, la ville est devenue celui du roman social moderne. 
les de prévention des aphore centrale pour ariale. Au-delà de ses à décrire suivant les oins comme objet de

dales entre les récits ilosophique (l'interin historique des apla connaissance du e d'attitudes et de ci pour notre part à ronotopes", suivant iis à la plume de tre sur leur caractère

onnaissance acquise ie universitaire et la sociologique et du tion industrielle a-tklassen in England) ays Noir anglais, à u laboratoire urbain testamentaires (Die ism as a Way of Life Theodore Dreiser, fameux Manhattan scopique représente ittérature plutôt que toix, par exemple le oyce, mais on serait Idants scientifiques orique sur la ville snt pour des raisons on à un atelier local matiquement les points nodernité urbaine ou le de recherche), mais aussi parce que cette métropole méditerranéenne dont l'image s'est faite (ou surfaite) sur l'échange entre les cultures du monde a inspiré nombre d'écrivains, passagers et locaux, et attire particulièrement aujourd'hui les sciences sociales et les cinéastes ${ }^{6}$. La lecture transversale de quelques ouvrages témoins récemment parus précise l'analyse comparée.

Manchester au siècle dernier, Chicago-New York au début du siècle et Marseille à la fin, soit trois terrains préalables à d'autres comme ceux de Tokyo ou de Dakar si l'on voulait au moins prétendre à quelque dimension anthropologique. Soit donc aussi trois chronotopes successifs à confronter sous le double rapport de leur spécificité narrative et de leur contribution à la connaissance urbaine. D'ores et déjà, leur sélection même fait peser sur chacun de fortes présomptions : entre Engels et Dickens se jouerait la question du réel et de l'imaginaire ; entre Simmel et Dos Passos (via l'Ecole de Chicago), celle de l'esthétique des formes de la vie urbaine; et entre les recherches et les romans sur Marseille, celle des modalités concurrentes d'expression de la dialectique entre le lieu particulier et l'histoire générale.

\section{"Des faits, des chiffres et non des sentiments !"}

Cuvre de jeunesse par laquelle l'auteur rend compte de son premier stage au pays de la révolution industrielle, La situation des classes laborieuses en Angleterre (1845) est à juste titre considérée comme un modèle pionnier d'enquête urbaine qui cherche à expliquer les raisons de ce qu'elle décrit. Comment comprendre en effet cette concentration brutale et sans précédent d'hommes et de briques autour de gigantesques manufactures, puits de mines et cheminées, ce voisinage inconcevable du luxe et de la misère, cet amassement de "spectres blêmes, poussés en hauteur, à poitrine étroite, figures atones et vidées de toute énergie" qui hantent les faubourgs délabrés du noir Manchester ou Birmingham ? Autant d'exilés de la campagne, là où leurs parents, paysan-ouvriers "soumis, modestes et naîfs" se contentaient d'une "vie paisible de végétaux", est-il précisé non sans condescendance pour le monde rural finissant. Au fur et à mesure qu'il parcourt le nouvel empire industrialo-urbain, qu'il inspecte ses taudis pestilentiels, qu'il rapporte les statistiques de criminalité et de mortalité, qu'il exhume d'un procès verbal de police le cadavre emplumé, faute de vêtements, d'une jeune mère agonie de faim et précocement rongé par la vermine (glacial effroi qui rappelle aujourd'hui la brûlante actualité des images de détresse en Afrique), l'enquê. teur avance les raisons d'un tel chaos ou "meurtre social". L'accumulation

6. Comme en témoigne le dossier "Ville et cinéma" du numéro 86 d'Espaces et Sociétés
(1996). 
capitaliste est au principe économique de la concentration spatiale ; l'immigration des prolétaires irlandais, ces "ivrognes bestiaux et insouciants" qui errent en loques, ne se nourrissent que de pommes de terre et dorment entassés dans des soutes à cochons, offre une armée de réserve qui réduit le coût de la main d'œuvre ; la prétendue libre concurrence ou la guerre de tous contre tous chère aux darwiniens sociaux, ne profite qu'à la minorité des propriétaires des moyens de production. Engels dénonce les possédants qui se dédouanent à peu de frais en accusant la ville de tous les maux. Car pour lui, cette ville qui isole les hommes et les ravale au rang aveugle de bêtes ou de machines, est aussi le lieu du rassemblement des travailleurs et de leur formation en classe consciente de sa vocation historique. Bel exemple du jeu de l'imaginaire et du réel, où la ville devient plus qu'un décor comme l'illustre de son côté Dickens dans Les temps difficiles (1854), roman de maturité et de réprobation du capitalisme.

Pandémonium d'usines fumantes, de mines actives ou effondrées, de chemins de fer, de décharges, de taudis et de maisons de maître isolées, Coketown (alias Manchester ou Birmingham) ponctue le récit comme un chœur de tragédie. Résumons l'intrigue...

Thomas Gradgrind, bourgeois vivant aisément de ses rentes à Coketown, professe l'utilitarisme dans les clubs, les écoles et bientôt au Parlement : "Des faits ! des chiffres et non des sentiments". Doctrine inflexible qu'il inculque de force à ses enfants, Louisa et Tom. Gradgrind impose à Louisa le mariage de raison avec le vieux et brutal Bounderby, principal industriel et banquier de la ville. Mais Gradgrind recueille aussi une jeune saltimbanque abandonnée, Sissy, contre l'avis de Bounderby. Ce dernier chasse de la fabrique son ouvrier préféré, Blackpool, qu'il soupçonne d'être un meneur. Louisa (Madame Bounderby) ose s'apitoyer sur le triste sort de l'ouvrier et l'aider par l'intermédiạire de Rachael, vertueuse ouvrière qui aime Blackpool. Tom, le cadet de Louisa, est devenu dans l'ombre un piètre joueur criblé de dettes ; il cambriole la banque Bounderby qui l'emploie en faisant accuser Blackpool, lequel, réprouvé par les travailleurs pour complicité avec le patron, doit fuir la ville. Pour renforcer son parti conservateur, Gradgrind fait venir à Coketown le noble Harthouse. Une idylle se noue entre ce bel aventurier et Louisa. Celle-ci, affolée par cette passion, trouve refuge chez son père, qui la recueille avec remords. Louisa, Sissy et Rachael font cause commune pour disculper le brave Blackpool qu'elles retrouvent agonisant au fond d'un puits de mine abandonnée. Reconnu coupable de ses délits, Tom le garnement fuit en Amérique, par l'entremise de Sleary, l'habile et chuintant patron du cirque où naquit Sissy ; là-bas, une fièvre l'emportera bientôt. Sćparée de son mari, Louisa s'occupe de folklore enfantin ; le sanguin 
centration spatiale ; l'imrestiaux et insouciants" qui le terre et dorment entassés rve qui réduit le coût de la I guerre de tous contre tous norité des propriétaires des Jants qui se dédouanent à Zar pour lui, cette ville qui bêtes ou de machines, est le leur formation en classe lu jeu de l'imaginaire et du lustre de son côté Dickens rité et de réprobation du

ictives ou effondrées, de raisons de maître isolées, nctue le récit comme un

le ses rentes à Coketown, ientôt au Parlement : "Des $\approx$ inflexible qu'il inculque upose à Louisa le mariage pal industriel et banquier ine jeune saltimbanque Ce dernier chasse de la sçonne d'être un meneur. triste sort de l'ouvrier et 'rière qui aime Blackpool. un piètre joueur criblé de nploie en faisant accuser pour complicité avec le sservateur, Gradgrind fait le se noue entre ce bel sion, trouve refuge chez sy et Rachael font cause $s$ retrouvent agonisant au able de ses délits, Tom le ary, l'habile et chuintant :vre l'emportera bientôt. e enfantin ; le sanguin
Bounderby meurt d'une crise cardiaque ; Sissy trouve le bonheur conjugal et maternel. Enfin, Gradgrind retiré du monde, se repend des ravages causés par
son parti pris pour la raison utilitaire.

Coketown-la-noire rythme donc le drame sous les traits d'une machine infernale ("A l'horizon, le piston des machines à vapeur montait et descendait monotonement comme la tête d'un éléphant fou de mélancolie") ou de labyrinthe miséreux dans lequel le monde se brouille (le "brouillamini" que l'infortuné Blackpool invoque machinalement). Mais ce "chaudron graisseux l'été" brasse aussi le destin des hommes. A la différence des bourgeois d'Engels qui côtoient la misère sans la voir, ici les classes se mêlent dans le conflit qui oppose le sentiment à l'intérêt. Bounderby, le pire des patrons, est un ancien ouvrier ; la riche Louisa devient l'amie intime de la pauvre Rachael ; les saltimbanques, catégorie sociale inconnue d'Engels, dénouent l'intrigue en bon "embrayeurs paratopiques" (MAINGUENEAU, 1993). Mais si le repentir final de Gradgrind atteste qu'un grain de sable s'est irremédiablement introduit dans les rouages du capital et de sa raison utilitaire, les destins perdus de Louisa, Tom, Rachael et Blackpool rappellent que les éléphants fous de mélancolie n'arrêtent pas pour autant de broyer l'humanité. L'envol prophétique qui clôt l'analyse d'Engels n'est pas si simple à Coketown où les leaders ouvriers corrompus accusent injustement l'un des leurs. Tout se passe finalement comme si le romancier, grâce à sa fiction pénétrante, rappellait le savant révolutionnaire à l'ordre complexe de la réalité, dont la ville chaotique de l'ère industrielle naisssante représente le plus évident symbole. Triste fable qui peut aussi valoir pour les illusions réalistes à venir du "roman expérimental" de Zola.

\section{Babel haletante}

Avec Chicago, on entre de plain-pied dans le destin métropolitain du siècle, lorsque populations, marchandises et cultures du monde se trouvent attirées et propulsées par de gigantesques villes qui croissent à vive allure. Dès 1903, Simmel voit de Berlin que cet emballement urbain précipite le détachement individuel des anciennes solidarités communautaires et des référents universels issus des Lumières. La mobilité et la relativité des identités devient la règle de l'individuation. L'argent, la montre ou la bourse consacrent l'abstraction du temps et l'équivalence des valeurs. Les grands boulevards, magasins ou hôtels forment l'empire de la mutuelle réserve, de l'excitation des sens, de l'attitude blasée ou du simulacre. L'étranger en sés aventures langagières ou juridiques s'impose là comme la principale figure de cette citadinité où chacun devient l'étranger de l'autre, et notamment de soi. 
metme. Plus de trente années plus tard, Wirth (1938) tente de tirer le bilan de 1'ekceptionnelle entreprise d'enquêtes sur l'urbanisation des mœurs qu'a été lo fameux département de sociologie de l'université de Chicago. Y a-t-il dans tout cela matière à théorie sociologique ? s'interroge-t-il avant de convenir qu'il faut d'abord ordonner les idées : la ville s'appréhende comme structure matếrielle, organisation sociale et système d'attitudes. A mettre en relation ces trois niveaux, le sociologue, observateur direct de la mosaïque chicagoane, peut conclure sur un double processus à l'œuvre : l'individuation par l'éclatement et l'enchevêtrement des rôles sociaux (chaque citadin est tout à la fois et alternativement usager, passant, citoyen, salarié, parent, jeune, vieux, etc.) et le nivellement par les espaces de droit commun (Etat, municipalité, école, rue, etc.). La métropole moderne est le manifeste dynamique de cette tension à travers les mille et une manières par lesquelles les citadins définissent entre eux leur situation, de coopération ou de conflit, d'alliance ou de séparation. Autant dire que les scènes d'hésitations et de malentendus où affleure l'incongruence des statuts entre les personnes vont occuper le terrain au grand dam de l'explication du phénomène urbain par les grandes instances - perspective qui s'imposera près d'un demi-siècle plus tard en France.

On a pu voir dans Sister Carrie, premier roman de Theodore Dreiser au tout début du siècle, une anticipation littéraire des idées des sociologues de Chicago (PÉTILLON, 1991). Carrie, jeune fille du bouseux Middle West et immigrée d'origine hollandaise, arrive à Chicago pour tenter d'y faire sa vie. Cette ville est en 1900 un gigantesque chantier où tout est possible. Carrie y connaît d'abord l'humiliante quête du travail et l'exploitation à la chaîne. Mais, jolie et intelligente, elle fait la rencontre d'un commis voyageur qui la sort de l'usine et lui fait goûter aux plaisirs de la ville. Hurstwood, un respectable directeur de boîte de nuit chic s'éprend d'elle au point de commettre l'irréparable en volant la caisse pour amener de force Carrie à New York vivre une autre vie. Ville plus riche et impénétrable que Chicago, New York devient alors la scène de la lente déchéance de Hurstwood rongé par le remords et qui, d'une affaire foireuse à l'autre, sombre dans la clochardise. Mais New York est aussi la scène de l'irrésistible ascension de Carrie comme actrice de music hall. Devenue vedette adulée par cette ville qui la fascinait naguère, Carrie n'a pas trouvé pour autant le bonheur.

Ici la description détaillée du manège urbain (où se mêlent robes de marque et chaussures usées, limousines rutilantes et tramways poussifs, confidences romantiques et querelles domestiques) importe d'autant plus que les personnages s'attachent plus aux choses qu'aux êtres. Miroir de tous les désirs, la ville réfléchit aussi l'illusion sociale : Hurstwood y sombre corps et biens; Carrie en fait une expérience douce-amère. Certes les battements de 
: de tirer le bilan de des mœurs qu'a été licago. Y a-t-il dans 1 avant de convenir de comme structure I mettre en relation ;t de la mosaïque vre : l'individuation taque citadin est tout llarié, parent, jeune, nmun (Etat, municiifeste dynamique de squelles les citadins conflit, d'alliance ou $t$ de malentendus où int occuper le terrain les grandes instances is tard en France.

Theodore Dreiser au $\mathrm{s}$ des sociologues de seux Middle West et enter d'y faire sa vie. est possible. Carrie y loitation à la chaîne. nmis voyageur qui la ville. Hurstwood, un d'elle au point de de force Carrie à New le que Chicago, New irstwood rongé par le z dans la clochardise. sion de Carrie comme : ville qui la fascinait

se mêlent robes de $t$ tramways poussifs, orte d'autant plus que es. Miroir de tous les ood y sombre corps et rtes les battements de cour et de cils de Carrie, ses sentiments troublés ou ses moments d'hésitation Illustrent à merveille les réflexions simméliennes sur l'excitation des sens ou la division urbaine de soi, mais l'arrière-plan moral (l'argent ne fait pas le bonheur) et la facture réaliste de ce roman social nous incite, tout en restant à New York, à prendre Manhattan Transfer (1925) comme le véritable roman du phénomène urbain tel qu'il interroge les sociologues de Chicago?.

Onomatopée haletante de la principale gare de triage de New York, manhat-tan-trans-fer, l'œuvre phare du jeune Dos Passos ("Le romancier de notre temps" selon J.-P. Sartre), accorde d'emblée la forme narrative à son propos : des centaines de séquences de vie urbaine qui mettent en scène l'existence simultanée d'une cinquantaine de citadins au cours du premier quart de siècle. Ici l'intensification de la vie nerveuse chère à Simmel est rendue par l'écriture de la ville en plans coupés, à la manière des peintres cubistes. Couleurs, bruits, et odeurs construisent le puzzle urbain (raies de soleil, sirènes du port, enseignes lumineuses, sueurs froides, chevelures féminines, gargouillements d'estomacs, vapeurs d'essence, etc.) à l'image de la trame fragmentaire des intrigues humaines. Banales histoires du succès ou de l'échec en amour et en affaires que les protagonistes vivent en termes de "chance" ou de "guigne". Mais derrière ces petites histoires de fils de comptables ou d'épiciers qui deviennent acteurs ou journalistes ${ }^{8}$, la ville-port se fait aussi l'écho du grondement du monde : la première guerre mondiale, le krach boursier et la levée des masses en Russie. Au fil des pages, les séquences de vie semblent aimantées par l'épuisement des corps (les sveltes danseurs d'hier s'attablent lourdement) et par les espérances déçues (la fameuse "génération perdue"). Colosse aux pieds d'argile comme Babel que Dieu aurait détruit en sept minutes, New York reste cependant pour ses habitants ou ses passants "aussi insupportable qu'indispensable".

En revenant aux régimes de vérité propres à chaque genre narratif, tout se passe comme si, au fur et à mesure qu'on avance dans le siècle, le roman social prenait toujours plus le souci de sa forme, délaissant par là-même aux sciences sociales montantes l'impératif de véridicité issu du moment naturaliste. $\mathrm{Ce}$ détachement instaure ainsi une tranquille complémentarité entre le roman et la sociologie dans la représentation du monde ou de la ville. Tranquillité qui n'est que d'apparence depuis que le positivisme s'effondre, que l'anthropologue se fait écrivain et que le romancier lit et traduit parfois les sciences humaines. On

\footnotetext{
7. Dans son ouvrage récent, dont on a pris connaissance juste avant la publication de cet article, P.Saint-Arnaud (1998) montre pour sa part que J. Dos Passos donne toute la mesure de son talent stylistique et sociologique dans la trilogie U.S.A. (The $42 d$ Parallel, Nineteen Nineteen, The Big Money ) postérieure à Manhattan Transfer.

8. Petits-enfants probables de paysans immigrés d'Europe du Nord, voire arrière-petits-enfants des prolétaires irlandais d'Engels.
} 
peut aisément supposer que cette concurrence discrète entre genres narratifs et disciplines accroisse les effets de distinction autant que les essais d'hybridation. De ce point de vue, la ville comme métaphore de la condition humaine telle que l'on vient de l'entrevoir entre Berlin et New York se confirme aussi comme thème d'analyse des jeux de langage contemporains.

Le caractère à la fois insupportable et indispensable du New York de Manhattan Transfer et les regards inquiets ou désabusés que ses habitants ou passagers jettent sur l'orage qui menace le monde nous conduisent tout naturellement vers Marseille dont la légende est faite d'attirance et de rejet ainsi que de rencontres entre le port et le monde. Cette ville fait récemment l'objet, comme on l'a dit, d'un investissement sans précédent d'études, de réflexions ou de récits qui interrogent ou mettent en scène "l'identité marseillaise". Rien qu'en 1995 (année d'élections municipales), on ne recense pas moins d'une dizaine d'ouvrages sur l'histoire, la population ou les lieux de la ville et deux romans policiers ${ }^{9}$. A les feuilleter, on s'aperçoit vite que cette effervescence s'explique globalement par le travail que les métropoles européennes engagent sur leur image et localement par le sentiment persistant de la désorientation symbolique et économique consécutive au deuil post-colonial. Ville "sans nom" et "sans monument", Marseille prend alors facilement l'allure mythique d'un personnage collectif dont la mauvaise réputation nationale est à déconstruire pour se ressaisir dans le nouveau cours du monde. C'est dire ici combien l'avenir attend que les trous de mémoire se comblent.

\section{La conjugaison des temps}

Dans cette anamnèse savante le regard des écrivains devient précieux (de GAUDEMAR, 1985). De la "Porte de l'Orient" du premier Empire à la "Villemonde" d'aujourd'hui, l'histoire littéraire de Marseille est traversée par la tension entre l'ici et l'ailleurs. Les grands écrivains voyageurs du siècle dernier,

\footnotetext{
9. Ouvrages sur Marseille parus en 1995 :

C. Bromberger, Le match de football, ethnologie d'une passion partisane à Marseille, Naples et Turin, Paris, Maison des Sciences de l'Homme ;

P. Carrèse, Trois jours d'engatse à Marseille, Paris, Fleuve Noir ;

P. Echinard, Marseille au quotidien, Marseille, Laffitte ;

J.-C. Izzo, Total Khéops, Paris, Gallimard-Série Noire ;

J. Kéhayan, Marseille, Paris, Hazan ;

A. Médam, Blues Marseille, Marseille, Laffitte ;

A. Tarrius, Arabes de France dans l'économie mondiale souterraine, La Tour d'Aigues, Aube ;

E. Témime, Marseille-Transit: les passagers de Belsunce, Paris, Autrement ;

J. Viard, Marseille, une ville impossible, Paris, Payot;

R.-P. Vigouroux, Quelle est ta ville?, Paris, Grasset.

Notons également une récente anthologie littéraire sur cette ville (1997): Agostini, J et Forno,

Y. Les écrivains et Marseille, Marseille, Laffitte.
} 
e entre genres narratifs et $t$ que les essais d'hybri: de la condition humaine ${ }_{v}$ York se confirme aussi porains.

isable du New York de usés que ses habitants ou te nous conduisent tout l'attirance et de rejet ainsi le fait récemment l'objet, d'études, de réflexions ou imarseillaise". Rien qu'en pas moins d'une dizaine le la ville et deux romans : effervescence s'explique iéennes engagent sur leur désorientation symbolique Tille "sans nom" et "sans mythique d'un personnage construire pour se ressaisir ien l'avenir attend que les

ains devient précieux (de remier Empire à la "Villeieille est traversée par la syageurs du siècle dernier,

partisane à Marseille, Naples $\mathrm{r}$;

aine, La Tour d'Aigues, Aube ; ; Autrement ;

le (1997) : Agostini, J et Forno, de Lamartine à Flaubert en passant par Stendhal, soulignent le grouillement tumultueux, l'excès, l'exubérance, la démesure, la désinvolture du lieu comme s'il s'agissait d'autant de signes avant-coureurs de leur rencontre avec l'Autre lointain. Ces traits exotiques se croisent avec la veine folklorique d'écrivains locaux comme Joseph Méry ou Horace Bertin. En traits pleins ou creux, la cité devient, au cours de la première partie de notre siècle, un objet d'attirance et de rejet sur fond de sourde tension entre l'écrivain "enfant du coin" et le "parisien" (les Dumas, Rostand, Zola, Suarès, Giono ou Pagnol ayant été l'un ou l'autre selon les moments). Métropole antique sans autre monument que celui de son style populaire fait de fierté et d'humour mais aussi de trafic secret, cette ville qui vit dans l'instant fascine un Cendrars vagabond parce qu'elle demeure "insaisissable". Depuis les transitaires exilés de la seconde guerre mondiale (Walter Benjamin, Anna Seghers) jusqu'aux dernières vagues de migrants du Maghreb, d'Afrique ou d'Asie, l'ici rejoint enfin l'ailleurs. Pour les nouveaux romanciers de passage (Le Clézio, Tournier), les prunelles de Lalla ou d'Idriss, jeunes migrants d'Afrique du Nord, reflètent l'éclat lointain de leur désert natif sur les rues sombres de la vieille ville.

Le roman et son commentaire se conjuguent donc ici avec le tournant narratif des sciences sociales. Cantonnée avant guerre à quelques monographies géographiques et économiques sur la ville-port, la recherche urbaine locale s'étoffe progressivement après guerre autour d'enquêtes sur la division sociale de l'espace et la centralité, puis se densifie et se polarise doublement à partir des années quatre-vingt autour d'une socio-anthropologie des migrations et d'une histoire économique et sociale de la ville-monde (ZALIO, 1997). Dans cette dernière période, la ville n'est plus un terrain parmi d'autres de description de la vie ouvrière ni un cas spécifique de médiation entre le pouvoir local et le mode de production capitaliste ; elle devient un objet en soi de connaissance universelle qui s'éprouve dans le récit généalogique ou phénoménologique du lieu.

De ces dernières années, si généreuses en publications, on a retenu trois exemples significatifs pour notre enquête : la thèse de Marcel Roncayolo, Les grammaires d'une ville (1996); l'essai de Alain Medam, Blues Marseille (1995) ; et les deux "polars"10 de Jean-Claude Izzo, Total Khéops (1995) et

10. On reprend ici la distinction faite par J.-N. Blanc (1991) entre le roman policier qui narre une énigme de manoir dans la langue de l'élite et le polar qui raconte le labyrinthe urbain dans la langue du peuple, "récit d'un monde brutal, rapide, orgueilleux et impitoyable, où l'acier, le fer, le béton, l'asphalte, l'automobile et la vitesse règnent en maîtres. C'en est fíni des gazons verts, du bruit léger du vent dans les frondaisons et de la douce tiédeur des coins de cheminée qui convenaient si bien aux scènes classiques des romans à l'anglaise où un lord désinvolte résolvait des mystères en tirant sur sa pipe. Dans le polar, la ville est désormais partout, jusque dans l'écriture." Sur l'apparition récente d'un polar à la marseillaise, voir : M. Samson,"Marseille, hérö̈ne de polars", in Le Monde, 6. 12. 1997. 
Chourmo (1996). Autant dire qu'entre la thèse de morphologie sociale sur la genèse des structures urbaines à Marseille, fruit volumineux et dense d'une quarantaine d'années d'analyses méthodiques dans la tradition savante d'Halbwachs, l'essai socio-phénoménologique de poétique de la ville, enlevé en deux ou trois ans dans la lignée prestigieuse des Sansot et Bachelard, et les polars humanistes qui fouillent le "côté obscur de la planète Mars(eille) ${ }^{11}$ " dans la veine populaire des Léo Malet ou Dashiell Hammett, s'étend un large champ sémantique bipolarisé entre vérité et fiction, cercle d'initiés et grand public. Précisons qu'il s'agit dans le premier et le dernier cas de natifs de Marseille (migration italienne), qui publient chez un éditeur parisien et dans celui du milieu d'un voyageur portraitiste de métropoles (Montréal, New York, Naples, Jérusalem), par ailleurs socio-analyste de la diaspora juive, qui publie chez un éditeur marseillais.

Pour le géographe Roncayolo, Marseille est un cas exemplaire d'étude de la division sociale de l'espace, thème universel et central de l'ouvrage. En écho déférent à la fameuse thèse d'Halbwachs qui démontra magistralement au début du siècle combien la transformation immobilière du Paris du Second Empire à la Belle Epoque devait si peu au choix rationnel des investisseurs et des administrateurs, le normalien administre ici la preuve par les variations concomitantes du peuplement, des investissements économiques et des formes urbaines, qu'un modèle libéral spécifique résiste à toute planification depuis la première grande extension urbaine au sud (autour de l'arsenal) initiée en 1666 par les intendants de Louis XIV. La double tradition locale du négoce portuaire et de la petite rente immobilière expliquerait la pusillanimité urbanistique et esthétique des édiles, l'échec de l'haussmannisation au nord de la ville, sa division sociale entre le nord populaire et le sud bourgeois et aujourd'hui le déficit de centralité dans une région urbaine multipolaire. Toute proportion gardée, cette généalogie structurale révèlerait un principe de développement local qui ressemblerait plus à celui de Londres qu'à celui de Paris, l'éternel adversaire. Tel est, très schématiquement, le principal effet de connaissance obtenu au prix de longues et scrupuleuses analyses de séries démographiques, de plans cadastraux et d'archives municipales. En toute rigueur, la démonstration durkheimienne se passe ici de toute évocation littéraire qui pourrait épaissir cet habitus urbain libéral qui résiste au temps et à l'Etat.

Dans son Blues Marseille, Medam fait au contraire son miel des romanciers locaux et de passage depuis un siècle avec lesquels il partage une même fascination pour cette cité "chaotique, tonitruante, suspecte et insaisissable". L'extrait suivant de son récit d'approche donne le ton.

11. "De la planète Mars...", album du groupe de rap marseillais IAM (1991). 
rphologie sociale sur la mineux et dense d'une $s$ la tradition savante tique de la ville, enlevé nsot et Bachelard, et les a planète Mars(eille) ${ }^{11}$ " mmett, s'étend un large sercle d'initiés et grand lernier cas de natifs de éditeur parisien et dans spoles (Montréal, New de la diaspora juive, qui

$s$ exemplaire d'étude de al de l'ouvrage. En écho intra magistralement au ère du Paris du Second nnel des investisseurs et reuve par les variations onomiques et des formes te planification depuis la 'arsenal) initiée en 1666 icale du négoce portuaire llanimité urbanistique et : au nord de la ville, sa urgeois et aujourd'hui le olaire. Toute proportion ncipe de développement celui de Paris, l'éternel al effet de connaissance z séries démographiques, En toute rigueur, la évocation littéraire qui au temps et à l'Etat.

: son miel des romanciers :Is il partage une même uspecte et insaisissable". in.

IAM (1991).

«Bruissements de voix. Bruissements d'images. Odeurs d'urine ou de poissons ? Descendre les escaliers de la gare Saint-Charles, chaque fois, c'est artiver comme un planeur sur la ville, la prendre de haut puis s'y laisser aller. Chair de ville. Chairs de passions. Odeurs d'huile. Fricassées. Vibrations. Visages-moteurs marqués par la ville, devenus cylindres ou culasses. Place du Marché des Capucins : des légumes vendus sous le regard d'une reine de pierre. Madame de Noailles, en fait. Marteaux piqueurs. Hassid courant. Cette suspension des voix propre aux marchés de plein air : qui parlent sans se parler, qui commercent. Murmures des ruisseaux d'affaires qui forment les fleuves économiques. La vitalité de Marseille tient à sa non-monumentalisation et à son innovation imperceptible, modeste, multiforme, colorée, et souvent "de mauvais goût" à vrai dire ! Il y en a pour tous les goûts : toutes les bourses, toutes les cultures, toutes les différences. Visages-cultures. Visages-différences. "Si tu veux qu'on en parle, on en parle" dit quelqu'un. L'accent d'ici, à nouveau, une façon de jeter la voix pour prendre position dans l'espace.»

Le visiteur de métropoles vient ici tenter de réduire le déséquilibre entre un trop plein de signifiés et un déficit chronique de signifiants. Pour cela, il emprunte les chemins de traverse entre le sensible et l'intelligible, parcourant la ville, sa presse et ses récits pendant une année pleine. L'année 1993 précisément, celle de la montée en gloire de l'OM et de sa descente aux enfers ; celle du "tapisme" naissant, aussi bruyant qu'incertain ; celle de la pire crise que le port ait connue avec le dernier conflit des dockers ; celle aussi de l'effervescence culturelle dans les friches industrielles. L'arpenteur trouve le lexème nature comme premier signifiant : "dénaturations", tout ce qui pourrit une cité par le bas, des ordures ménagères qu'une grève interminable entasse dans les rues nauséabondes à la lancinante chronique des dealers et des overdoses ; "surnaturalités", les menaces extérieures qui pèsent sur les citadins inquiets, du détraquement portuaire jusqu'à l'ombre noire du sida en lointain écho de la peste du Grand Siècle ; "artificialisations", les autoroutes, tunnels et autres équipements modernes qui dispersent le quotidien ; "naturalisations", le miracle continué à chaque vague migratoire d'une intégration locale respectueuse des différences. En conjuguant à plusieurs temps ces états de nature, l'auteur "pense l'hétérogène" pour comprendre qu'en cette année éprouvante, l'ancestral victimisme marseillais n'empêche pas quelques exploits technologiques et artistiques de renouer avec les succès commerciaux de l'époque portuaire et coloniale. Si la cité populaire des quartiers nord est loin de rejoindre la villa à piscine au sud, comme hier le cabanon s'opposait à la bastide, le roman de la ville s'écrit dans les interstices, les incongruences, là où le cosmopolite bouscule les communautés établies, là où se déploient les 
réseaux productifs qui lient Marseille à la Méditerrannée pour les uns et à l'Europe pour les autres. Mais l'accumulation des traits de cette ville "bourrée d'antinomies et d'évidences déniées" ne brouille-t-elle pas paradoxalement son image par excès de signifiés ou de stéréotypes ? C'est ce que semble se demander l'auteur, comme s'il se rendait compte qu'il n'avait fait que tourner autour de ce fameux "monument de commerce humain" qui remplace au jour le jour l'édifice manquant de la mémoire.

L'écriture romanesque s'arrête peut-être moins à la mythologie urbaine que l'essai de Médam, tant elle cherche à exprimer de façon originale un monde naturellement confus, une existence problématique ou un sentiment trouble. C'est ainsi que dans les polars marseillais de Izzo le récit dépasse les clichés sur la ville dont il ne se prive pas d'ailleurs. Total Khéops (alias "bordel immense", d'ampleur pharaonique) et Chourmo (alias "galère" à la phocéenne, c'est-à-dire un état d'esprit mêlant endurance et solidarité), expressions des rapeurs des quartiers nord, annoncent dans leur titre même que Marseille est bien plus que le décor des aventures suivantes..

Fabio, Ugo, Manu et Lolle, enfants des migrations italienne, espagnole, et gitane forment un groupe inséparable dans le Panier (le plus vieux quartier de Marseille) des années soixante. Misère, amour, grandes espérances et petite délinquance. Trente ans plus tard, Fabio s'est converti en flic social des cités populaires des quartiers nord, Manu et Ugo sont devenus truands et la belle Lolle, serveuse de bar louche. La guerre des gangs fait rage sur la ville, avec ses complicités policières et politiques sur fond de redistribution des cartes au sein de la Camorra. Manu puis Ugo sont abattus dans des règlements de compte qui les dépassent. Bravant sa hiérarchie corrompue, Fabio Montale se lance en privé dans l'enquête, au péril de sa vie. Vie solitaire et désabusée de "fonctionnaire du moindre mal" à laquelle le quinquagénaire ne tient plus tellement d'ailleurs, malgré ses conquêtes féminines, son goût pour le pastis, la poutargue, le jazz, le fado, la pêche à la palangrotte, le farniente au cabanon, etc. Les tueries que son enquête provoque dans le Milieu ne suffisent pas à venger Ugo, Manu et Leila, l'épatante étudiante beur atrocement violée et assassinée. Cliché final : Fabio démissionnaire enlace Lolle sauvée devant le spectacle majestueux du Vieux-port qui s'ouvre au petit jour sur la ville incertaine.

Dans Chourmo, Fabio Montale coule une douce et pagnolesque retraite anticipée dans son cabanon de pêcheur des Goudes (quartier excentré). Mais il doit quitter à regret sa palangrotte pour s'embarquer dans une nouvelle enquête justicière (c'est son "chourmo" à lui). Face aux puissances complices de la Mafia, des groupes armés islamistes et des éléments fascistes de la police affiliès au Front National, il réussit à venger sa belle cousine Gélou après être 
Inée pour les uns et à de cette ville "bourrée e pas paradoxalement z'est ce que semble se n'avait fait que tourner " qui remplace au jour

la mythologie urbaine de façon originale un tique ou un sentiment zzo le récit dépasse les s. Total Khéops (alias mo (alias "galère" à la Jurance et solidarité), t dans leur titre même suivantes..

italienne, espagnole, et a plus vieux quartier de les espérances et petite en flic social des cités enus truands et la belle it rage sur la ville, avec istribution des cartes au ans des règlements de npue, Fabio Montale se olitaire et désabusée de uagénaire ne tient plus son goût pour le pastis, igrotte, le farniente au ns le Milieu ne suffisent beur atrocement violée xce Lolle sauvée devant u petit jour sur la ville

et pagnolesque retraite quartier excentré). Mais quer dans une nouvelle Ix puissances complices ints fascistes de la police zousine Gélou après être passé plusieurs fois entre la vie et la mort. Trois cadavres d'innocents au départ et des dizaines de méchants éliminés à l'arrivée. Marseille et la police rếpublicaine résistent quand même à leur propre destruction.

A la différence des polars baroques de Montalban sur Barcelone, auxquels Izzo a manifestement emprunté la figure centrale du privé sybarite et désabusé (Pepe Carvalho), l'intrigue se donne ici à la première personne, celle de Fabio, dans le langage simple et coloré du narrateur local au contact facile. L'argot local des dialogues ("vé, fend de pute, cagole" et autres "engatse") mais aussi le style indirect libre du narrateur témoignent d'un milieu indissociablement géographique et social. Par Fabio interposé, Izzo fait donc parler Marseille plutôt que de gloser sur son identité populaire - antique procédé depuis Pétrone, comme l'a montré Erich Auerbach (1946). Plongeant dans "ce chaudron où bouillotte le plus étonnant coulis d'existence humaine qui se puisse imaginer, aromatique et rutilant" (cliché de Gabriel Audisio, écrivain marseillais que Fabio affectionne), l'enquête policière fait circuler les images entre les rues, les places ou les bars sous contrôle clanique et les artères marchandes nouvellement piétonnisées qui détournent le "thalweg social" (Roncayolo) de l'ancienne Canebière. Partagé dans son "chourmo" entre l'engagement et la résignation, l'activisme et l'attentisme, le héros révèle l'intimité d'une ville sujette à la tragédie et à la nostalgie. Son combat désespéré contre les tueurs de la Cité transculturelle (le Milieu, le racisme ou l'intégrisme ici coalisés) est aussi celui pour la survie d'une certaine culture méditerranéenne qui a l'art de méler le rire au sérieux et l'indolence au dynamisme, seule issue commune pour surmonter les nouveaux "temps difficiles" qui menacent. En somme, Total Khéops et Chourmo offrent, avec la cerise rap sur le gâteau, les signifiants que Medam appelait de ses vœux pour conjuguer le passé avec le futur, ainsi que l'exige le présent de la ville.

\section{L'arc et la lyre}

Nous voilà donc en présence de trois villes-époques dont l'agencement peut faire tableau : Manchester ou Birmingham pour l'industrialisation du monde au milieu du siècle dernier, Chicago ou New York pour sa métropolisation au début de ce siècle, Marseille pour sa symbolisation aujourd'hui. Chacun de ces chronotopes condense une image de la ville contemporaine et un régime de rapports entre science et littérature.

A Manchester, la ville est au centre du drame de la modernité, celui qui aliène l'homme sensible à la machine du capital et de la raison instrumentale ; à Chicago, la ville devient un phénomène universel de coexistence sociale à expliquer, comprendre, maîtriser ; à Marseille, elle s'impose comme mémoire 
d'un espace social local traversé par les mouvements de l'économie-monde. Dans le premier cas de figure, le roman se fait l'émule de l'enquête scientifique ; poussée jusqu'à son terme (socio) logique, la fiction narrative de Dickens apparaît rétrospectivement plus réaliste et moins réductrice que la prophétie autoréalisante d'Engels. Dans le second cas, le duo entre science et littérature joue d'autant plus de concert qu'il assume pleinement sa différence ; dans l'univers proliférant des formes qu'induit la métropole, les sociologues de Chicago rassemblent leurs observations méthodiques autour de concepts dynamiques, tandis que les romanciers d'avant-garde inventent le style simultané pour exprimer l'éclatement du monde. Dans le troisième cas enfin, les jeux de langage ou de frontières entre les énoncés savants, littéraires ou populaires s'accordent autant d'écarts entre eux que d'emprunts ; les métaphores sensibles peuvent aussi bien envahir l'essai sociologique que le parler peuple du polar venir au secours de l'abstraction savante pour exprimer le double mouvement de la ville-port qui à la fois résiste et s'ajuste au temps.

Les images de la ville comme les formes et les relations narratives qui composent notre tryptique prennent du relief en introduisant précisément cette dimension temporelle. C'est là un des effets seconds de notre petite exploration intertextuelle focalisée sur le rapport entre espaces et sociétés. Manifestement, pour Dickens comme pour Engels l'industrialisation du monde s'inscrit dans une conception orientée du temps, avec ses causes et ses effets mécaniques, ses contradictions et ses dépassements hégéliens ; tout drame humain, toute ville deviennent alors les sujets ou les agents heureux ou malheureux de l'Histoire. Avec les "socio-écologues" de Chicago et le simultanéisme de Dos Passos, le temps semble soudain éclaté dans les instants et à tout le moins réduit aux dimensions d'une génération, celle des migrants qui intègrent et font la grande ville puis celle des enfants de la crise de l'entredeux-guerres ; lạ fragmentation des durées, des rythmes et des mémoires règne sur la mosaïque urbaine. C'est peut-être à cette dissémination du sens dans le miroitement des clichés que résistent ceux qui sont censés penser la ville d'aujourd'hui ainsi que le montre l'exemple de Marseille. Grand "Etre unanime" du récit contemporain (ROMAINS, 1908), la ville insaisissable est à la recherche de ses marques, de ses signifiants dans la mémoire des lieux d'établissement ou de passage (d'où d'ailleurs la faveur récente du versant bergsonien de la sociologie urbaine d'Halbwachs) ${ }^{12}$. Science et littérature concourrent et interfèrent alors au discernement d'une ville façonnée par la pluralité du temps : temps-coquille d'un modèle libéral d'urbanisation, temps intergénérationnel de l'intégration des migrants, temps fluctuant des

12. Sur la piste ouverte par Halbwachs entre la conception subjectiviste de Bergson et la conception objectiviste de Durkheim, voir S. Mazzella (1997). 
its de l'économie-monde. nule de l'enquête scienti$\approx$, la fiction narrative de : moins réductrice que la as, le duo entre science et : pleinement sa différence nétropole, les sociologues iques autour de concepts garde inventent le style ins le troisième cas enfin, cés savants, littéraires ou s que d'emprunts ; les essai sociologique que le on savante pour exprimer siste et s'ajuste au temps. $s$ relations narratives qui iduisant précisément cette seconds de notre petite sntre espaces et sociétés. zls l'industrialisation du ups, avec ses causes et ses sements hégéliens ; tout ; ou les agents heureux ou gues" de Chicago et le in éclaté dans les instants ration, celle des migrants ants de la crise de l'entreythmes et des mémoires tte dissémination du sens qui sont censés penser la le Marseille. Grand "Etre ), la ville insaisissable est ans la mémoire des lieux aveur récente du versant ${ }^{12}$. Science et littérature une ville façonnée par la iral d'urbanisation, temps $s$, temps fluctuant des

conjonctures économiques lointaines, temps agité des affaires locales, temps présent, temps virtuel, etc.

Notre tableau en trois images de la ville et conceptions du temps ne peut certainement pas prétendre à un quelconque modèle de développement des tensions et contre-tensions entre l'arc du savant et la lyre du roman; ses traits successifs, aussi pertinents soient-ils, n'excluent pas leur cumul ni leur chevauchement ; sa facture occidentale reste à confronter à d'autres aires culturelles. On pourrait par exemple mettre en regard la définition de la ville japonaise comme "trace et matrice" (BERQUE, 1993) du lien entre les hommes, les signes et les choses, avec la manière dont le roman japonais contemporain use et s'écarte à la fois de l'esthétique occidentale. La rivière, symbole de l'impermanence du temps extrême-oriental, n'est-elle pas artificiellement restaurée au creux des gigantesques centres commerciaux de Tokyo, machines mondiales du temps comptable?

Enfin notre exploration schématique des régimes textuels de la ville appelle l'examen méthodique de leurs "pactes de lecture" respectifs, convergents ou divergents (VIALA, 1988). A travers eux, les cohérences logique ou esthétique recherchées et attendues correspondent à autant de formes de sociabilité scandées par l'histoire (CHAMBOREDON, 1986). La variation des valeurs et conditions attachées aux figures du créateur et du chercheur, à l'échelle d'une époque comme d'une vie d'auteur, en indiquerait sans doute le cours. Hier, à travers le projet naturaliste du siècle dernier, l'écrivain revendiquait un noble métier, le "génie solitaire" aspirait à devenir "travailleur solidaire" (CHEVREL, 1982) ; quelques années plus tard, au moment où ce projet est rapidement enterré par les diverses réactions "antiréalistes", Durkheim forçait l'entrée de la science dans la Nouvelle Sorbonne, consacrant l'échec d'une sociologie plus littéraire incarnée par Tarde. Aujourd'hüi, la séparation professionnelle des mondes cognitifs, normatifs et expressifs ne va pas, comme on l'a vu, sans quelques passerelles ou souterrains reliant ouvertement ou secrètement les genres, les disciplines, les styles et les statuts. La ville comme monde vécu (pour compléter notre allusion à Habermas) fait décidément la fortune de leurs aventureux passagers. 


\section{Références bibliographiques}

AUERBACH E. (1968), Mimésis, la représentation de la réalité dans la littérature occidentale, Paris, Gallimard, (1 ere édit. origin. 1946).

BAKHTINE M. (1978), Esthétique et théorie du roman, Paris, Gallimard, (1ère édit. origin. 1975)

BERQUE A. (1993), Du geste à la cité, Formes urbaines et lien social au Japon, Paris, Gallimard.

BIDOU-ZACHARIASEN C. (1997), Proust sociologue, De la maison aristocratique au salon bourgeois, Paris, Descartes et Cie.

BLANC J.-N. (1991), Polarville, Les images de la ville dans le roman policier, Lyon, Presses Universitaires de Lyon.

BROWN R. (1989), Clefs pour une poétique de la sociologie, Arles, Actes Sud, (1 ${ }^{\text {re }}$ édit. origin. 1977).

CAMPION P. (1996), La littérature à la recherche de la vérité, Paris, Seuil.

CHAMBOREDON J.-C. (1986), Production symbolique et formes sociales, De la sociologie de l'art et de la littérature à la sociologie de la culture, Revue Française de Sociologie, XXVII.

CHEVREL Y. (1982), Le naturalisme, étude d'un mouvement littéraire international, Paris, Presses Universitaires de France.

DE GAUDEMAR A. (1985), Marseille au gré des mots, La ville dans le roman français, in (coll.), Marseille ou le présent incertain, Arles, Actes Sud.

DE LA SOUDIÈRE M., PERROT M. (dir.) (1994), L'écriture des sciences de l'homme, Communications, $\mathrm{N}^{\circ} 58$.

DE SINGLY F. (1996), Le soi, le couple et la famille, Paris, Nathan.

GEERTZ C. (1996), Ici et là-bas, L'anthropologue comme auteur, Paris,

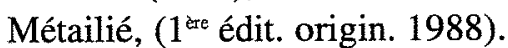

HEINICH N. (1996), Etats de femmes, L'identité féminine dans la fiction occidentale, Paris, Minuit.

KERBRAT M.-C. (1995), Leçon littéraire sur la ville, Paris, Presses Universitaires de France.

LEPENIES W. (1990), Les trois cultures, Entre science et littérature, l'avènement de la sociologie, Paris, Editions de la Maison des Sciences de l'Homme, (1 $1^{\text {te }}$ édit. origin. 1985).

LASSAVE P. (1997), Les sociologues et la recherche urbaine dans la France contemporaine, Toulouse, Presses Universitaires du Mirail. 
ion de la réalité dans la édit. origin. 1946).

roman, Paris, Gallimard,

urbaines et lien social au

'ogue, De la maison aristoet Cie.

'e la ville dans le roman n.

la sociologie, Arles, Actes

e de la vérité, Paris, Seuil.

bolique et formes sociales, la sociologie de la culture,

mouvement littéraire internce.

des mots, La ville dans le isent incertain, Arles, Actes

, L'écriture des sciences de nille, Paris, Nathan.

ogue comme auteur, Paris, :té féminine dans la fiction ar la ville, Paris, Presses ntre science et littérature, s de la Maison des Sciences rche urbaine dans la France :aires du Mirail.
MAINGUENEAU D. (1993), Le contexte de l'auvre littéraire, Paris, Dunod,

MAZZELLA S. (1997), La ville-mémoire, quelques usages de La mémoire collective de Maurice Halbwachs, Enquête, No4.

PÉTILLON P.-Y. (1991), O! Chicago : images de la ville en chantier, in (coll.), Citoyenneté et urbanité, Paris, Esprit.

RANCIÈRE J. (1992), Les noms de l'histoire, Essai sur la poétique du savoir, Paris, Seuil.

ROMAINS J. (1983), La vie unanime, Paris, Gallimard, (1 1908).

SAINT-ARNAUD P. (1998), Park-Dos Passos, regards croisés sur la modernité urbaine aux Etats-Unis, Toulouse, Presses Universitaires du Mirail et Presses Universitaires de l'Université Laval.

VIALA A. (1988), Effets de champ et effets de prisme, Littérature, $\mathrm{N}^{\circ} 70$.

ZALIO P.-P. (1997), Urbanités marseillaises : Marseille, terrain des sciences sociales, Enquête, $\mathrm{N}^{\circ} 4$.

ZÉRAFFA M. (1971), Roman et sociêté, Paris, Presses Universitaires de France.

Pierre Lassave est chargé de cours à l'Université Paris-X Nanterre. et corédacteur des Annales de la Recherche Urbaine, Secrétariat du Plan Urbain, Direction de l'Architecture et de l'Urbanisme, Ministère de l'Equipement, Tour Pascal B, 92055 - PARIS LA DÉFENSE CEDEX 4. 


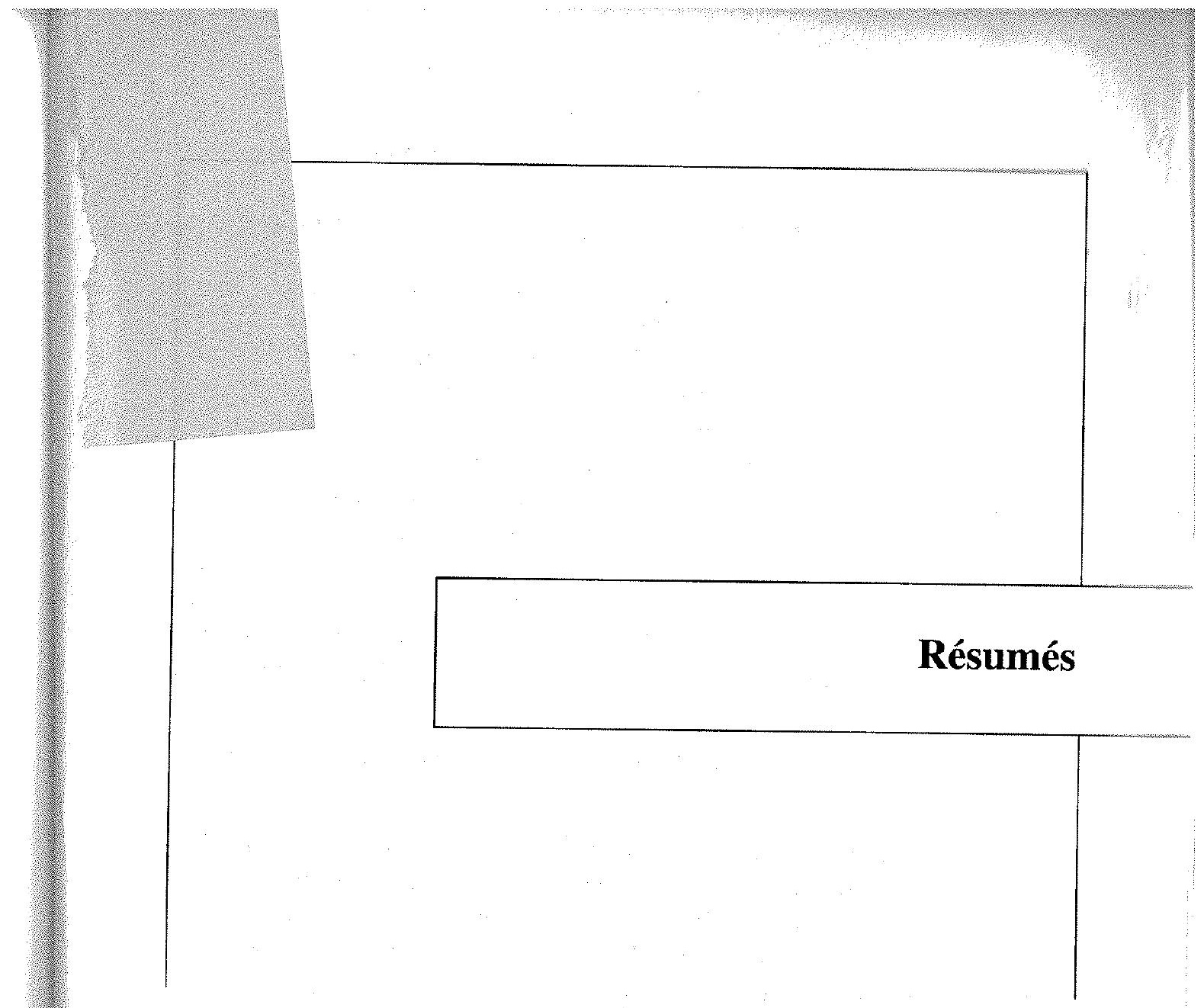

Pierre LASSAVE, La ville entre les lignes de la science et du roman.

Si la confusion des genres entre science et littérature ne semble pas pour demain tant la division des langues est au principe de la communication humaine depuis Babel, le jeu des correspondances, des équivalences, des emprunts réciproques entre la littérature au sens strict et la sociologie au sens large se fait plus pressant à reconnaître. A cet égard, trois villes-époques sont ici explorées : le Manchester de la première révolution industrielle, avec Dickens et Engels ; Chicago et New York au début du siècle, avec Simmel et Dreiser, Wirth et Dos Passos ; le Marseille contemporain, avec Roncayolo, Medam et Izzo. L'analyse met à jour trois configurations symboliques : d'abord l'émulation entre science et littérature pour exposer le drame de lindustrialisation du monde ; ensuite la dissociation ou la complémentarité entre les langages d'expression du phénomène métropolitain qui fragmente les identités ; enfin linteraction entre genres littéraires a la recherche de la mémoire plurielle des lieux. 
Jean-François ROULLIN, Ville et architecture écrite : de l'auteur au lecteur.

Aujourdhui, la constitution de la ville ou de l'architecture écrites en tant qu'objet permet de parler de champ identifié, où oeuvrent différentes disciplines. Les différents chercheurs, tout en légitimant ce champ, construisent et étudient cet objet du point de vue de leur discipline propre, par des approches sociologiques, géographiques, architecturales, littéraires, monodisciplinaires ou plus larges. Mais de ces approches, le lecteur est absent. C'est oublier qu'une cuvre est d'abord faite pour être lue par des non-spécialistes, que le premier destinataire nest pas le chercheur. L'article se propose d'introduire le lecteur dans le champ, comme sujet au travers de ses perceptions, et comme acteur parce qu'il est un des moteurs de l'histoire de la littérature. C'est alors non plus seulement se demander ce que la société dit à travers des textes, mais aussi ce qu'elle se dit à elle-même, dans le domaine de la ville et de l'architecture.

Catherine BIDOU-ZACHARIASEN, L'espace urbain comme ressource sociale dans le roman proustien.

Le propos de cet article est de montrer comment l'analyse sociale centrale du roman proustien, celle des rapports entre l'aristocratie et la bourgeoisie, traite la dimension spatiale. L'espace résidentiel, essentiellement urbain et parisien, y représente plus que le seul symbole d'une place ou d'un classement social. Il déborde également le cadre, la scène où se jouent les interactions sociales. Proust le construit aussi comme pouvant constituer une ressource à partir de laquelle s'élaborent des stratégies d'acteurs. En ce sens il est à la fois analyseur et opérateur des rapports sociaux.

\section{Bernard POCHE, Y a-t-il un roman lyonnais ?}

Cet article veut discuter, à partir de l'exemple de Lyon, l'existence d'une littérature rattachable comme telle à une ville précise. Une fois éliminée lobjection de lécrit régionaliste, cest-à-dire folkloriste, qui constitue une catégorie minoritaire, il apparaît que ce qui est significatif dans ces textes est l'expression dune société propre, et non la force des traditions ou la rémanence des symboles locaux. Si certains romans lyonnais de la première moitié du siècle parlent des questions concrètes qui agitent la ville, beaucoup d'autres traduisent simplement la sensibilité propre de cette société aux événements du temps. Lorsque cette littérature lyonnaise s'affaiblit, cest sous la pression d'un espace Imaginaire supposé illustrer toutes les formes possibles sous la figure de Paris. Il se crée alors en France un équivalent littéraire généralisé fictif, qui élimine le principe de réalité avec le prétexte d'exclure la particularité. 


\section{'e l'auteur au lecteur.}

cture écrites en tant ifférentes disciplines. nstruisent et étudient par des approches aonodisciplinaires ou C'est oublier qu'une listes, que le premier 'introduire le lecteur t comme acteur parce C'est alors non plus textes, mais aussi ce e l'architecture.

in comme ressource

ise sociale centrale du a bourgeoisie, traite la urbain et parisien, y I classement social. Il ctions sociales. Proust ce à partir de laquelle ì la fois analyseur et

yon, l'existence d'une e. Une fois éliminée $e$, qui constitue une tif dans ces textes est itions ou la rémanence emière moitié du siècle oup d'autres traduisent événements du temps. a pression d'un espace sus la figure de Paris. Il sé fictif, qui élimine le ité.
Christopher LUCKEN, Ecrire les fondations. Amiens et le Roman d'Abalane.

Si c'est à l'archéologue de retrouver les restes enfouis dune ville sous les pierres de celle d'aujourdhui, rien, sinon peut-être l'image, ne vaut l'écrit pour restituer une ville. La ville qui a disparu de la surface de la terre perdure en effet à travers l'écriture. C'est le cas pour de nombreuses villes qui au cours du Moyen Age, furent l'objet d'un genre de texte particulier, appelés parfois urbium laudes. A travers le Roman d'Abalane, transcrit (ou traduit) par un auteur anonyme, l'article illustre ce phénomène à propos de la ville d'Amiens.

\section{Jacques KATUSZEWSKI, La beauté des banlieues.}

Il n'est pas très facile de donner une signification sociologique au phénomène dit de l'esthétisation des banlieues, c'est-à-dire de la transformation de ce qui semble être "négatif" du point de vue social en une chose positive du point de vue esthétique. Deux théories sociologiques se sont emparées de la question pour en proposer un traitement qui me semble réducteur. D'une part, une théorie de type culturaliste : d'après elle, l'esthétisation des banlieues serait l'expression figée d'une époque qui se caractériserait par le pur jeu de signes, par le désintérêt pour le référent supplanté par un déchânement ininterrompu d'images qui tiennent lieu de réalité. D'autre part, une théorie de type structuro-fonctionnaliste qui traite l'esthétisation des banlieues comme une représentation marquée par l'appartenance sociale, celle par exemple des professions les plus enclines à saisir l'esthétique en toute chose.

Contre ces deux perspectives réductrices, je propose un point de vue pragmatique qui consiste à saisir l'esthétisation des banlieues dans sa diversité d'usages : une collection d'oeuvres (l'art des banlieues), un public (la banlieue comme terre d'accueil de l'oeuvre vraie), le plaisir d'un paysage (la beauté des banlieues), bref à prendre en compte l'affect, le corps, les activités, le plaisir.

Marian $\mathrm{ROCH}$, La spatialisation du social à l'épreuve de la mobilité : l'exemple de l'espace péri-urbain.

La forte mobilité spatiale individuelle qui caractérise les communes périurbaines permet un éclairage particulier du rapport entre formes sociales et formes spatiales. Tandis que la multilocalisation des espaces sociaux individuels conduit à un état d'opacité des rapports sociaux locaux, la capacité de valorisation de la mobilité spatiale fonctionne à la fois comme ressource et comme signe social dans les interactions individuelles. En permettant un encodage local des signes portés par les habitants aux modes de vie fortement individualisés, l'espace péri-urbain acquiert une certaine efficace socialisatrice, d'autant que la gestion de la vie collective locale devient un enjeu pour la définition des groupes sociaux locaux. 